\title{
Wireless power transfer in the presence of a conducting interface: Analytical solution
}

ISSN 1751-8725

Received on 20th October 2018

Revised 27th February 2019

Accepted on 4th March 2019

E-First on 5th April 2019

doi: 10.1049/iet-map.2018.5890

www.ietdl.org

\author{
Andrea Vallecchi ${ }^{1} \bowtie$, Ekaterina Shamonina ${ }^{1}$, Christopher J. Stevens ${ }^{1}$ \\ ${ }^{1}$ Department of Engineering Science, University of Oxford, Parks Road, Oxford OX1 3PJ, UK \\ 凶E-mail: andrea.vallecchi@eng.ox.ac.uk
}

\begin{abstract}
A comprehensive model of wireless power transfer (WPT) between a pair of inductively coupled loop resonators in the vicinity of a conducting slab is developed by combining electromagnetic (EM) theory with an equivalent circuit representation of the system. The model incorporates all EM interactions occurring between the coils both directly and through the slab. The design of an optimal WPT regime for two coils in the presence of a conducting slab with variable conductivity is demonstrated by exploiting the model to predict the coil detuning produced by the slab. It is further shown that the model also constitutes an effective tool to estimate the losses in the conducting material solely in terms of the parameters of the generalised equivalent circuit and to analyse the power flow in a WPT system. The validity of the analytical results is proved by comparison with rigorous full-wave simulations. The proposed analytical method can have particular relevance in the field of wireless charging of electric vehicles and WPT for supplying implanted medical devices and body sensor networks, where it can be instrumental for the design and optimisation of the system to be compliant with existing RF exposure safety levels.
\end{abstract}

\section{Introduction}

The ubiquitous diffusion of mobile communications and computing and the expanding utilisation of electric vehicles have stimulated increasingly greater interest in wireless power transfer (WPT) technology, due to its potential of rescinding the requirement for the last remaining wired connection to power and charge portable devices. Originally pioneered by Tesla one century ago $[1,2]$, nonradiative WPT depends on the magnetic coupling of resonant coils in the near field. Using antennas behaving substantially as magnetic dipoles provides the advantage that in the near field the magnitude of the associated electric field is considerably decreased; this in turn results in a weaker interaction with obstacles in the environment or biological substances, which are mostly dielectric materials, and improved transfer efficiency in short- and mid-range applications [3-5]. Major research efforts have been recently directed towards developing novel WPT systems with the capability of transporting energy over more extended ranges and with higher efficiency through the adoption of rely systems either employing intermediate resonators [6-8] or metamaterials [9-13]. The key factors to achieve high efficiency WPT, according to both theory and experiments, are the combined use of magnetic induction, tuned circuits, and operating at the resonance frequency. However, the improvement in power transfer efficiency (PTE) achievable by high $Q$ resonant WPT systems can have as a repercussion an increased sensitivity of the coils to the surrounding environment, which might be difficult to control dynamically [14].

In previously reported analyses and measurements, transmitter and receivers of WPT systems are most commonly assumed to be situated in free space. Yet, there are many applications that involve transferring energy into a receiver placed in more challenging electromagnetic (EM) environments; for example, in the context of the wireless charging of electric vehicles, the car body, usually metallic, is in close proximity to the WPT system [15]. The use of wireless supply to power implanted medical devices [16-20] and body sensor networks for healthcare and medical research [21] are other situations where taking into account the impact of the environment is particularly important. In fact, in these applications, substantial variations in load, coupling, and circuit parameters can be expected because the human body and biological tissues are moderately conductive; at the same time, there are strong requirements to maintain an effective power regulation to ensure that a constant power is delivered, as desirable [19], maximise transfer efficiency and thereby reduce heat dissipation in the coils, and provide compliance of WPT systems with human EM exposure limits [22].

As shown recently by both numerical simulations [23] and experiments [24], efficient WPT can be realised also in the presence of metallic plates either by relying on the symmetry of the configuration, e.g. when both the transmitting and receiving resonators have a metal plate in their vicinity, or otherwise, when a system is not spatially symmetric, through the regulation of the resonance frequency and impedance of the transmitter.

In this work, we aim at developing an analytical model of WPT in a system of inductively coupled resonators in the vicinity of a conducting slab. The method combines coupled coil theory [25] with the EM solution for the fields produced by a uniform current loop positioned above a conducting slab. The latter problem has been the subject of extensive investigation in the past in the field of electrical machines and, in particular, with reference to the characterisation of the magnetic fields surrounding the ends of turbo-generators [26, 27]. The magnetic field due to a single circular current filament above a conducting half space was derived in [28], and then extended to the case of a coil of arbitrary rectangular cross-section parallel to a conductor slab above a conducting half space of different conductivity in [29].

To simplify the exposition and without loss of generality, we will consider a WPT system comprising only two resonant conducting thin wire coils lying parallel to a conducting slab on the same side of it. It is noted that the proposed approach is more generally applicable to a system of multiple coils which can be arbitrarily located on either side of the slab. The exact field solution developed in [29], whose accuracy has been recently investigated in [30], is used to calculate the self- and mutual inductances of the coils in the presence of the slab, which are then incorporated into generalised coupled circuit equations for the coils. As a result, a complete description of the WPT system is accomplished which exactly takes into account all interactions contributing to controlling the power link, including the feedback from the receiving to the transmitting coil and the effect of the currents induced in the conducting slab. A preliminary discussion of this approach has been previously presented in [31].

The organisation of the paper is as follows. In Section 2, we formulate the problem and present the analytical solution. In 


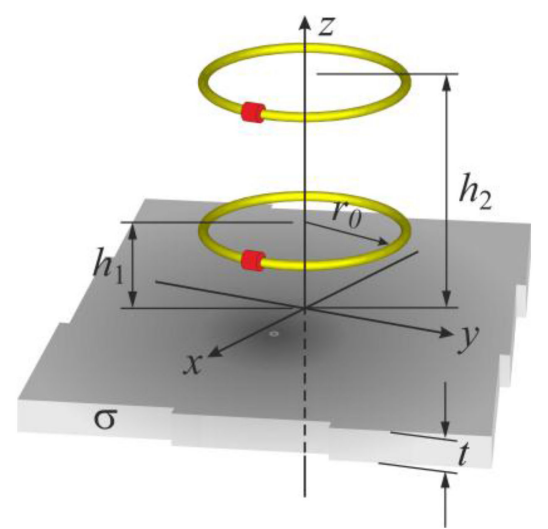

Fig. 1 WPT system consisting of two resonant circular coils above a conducting slab with conductivity $\sigma$ and thickness $t$

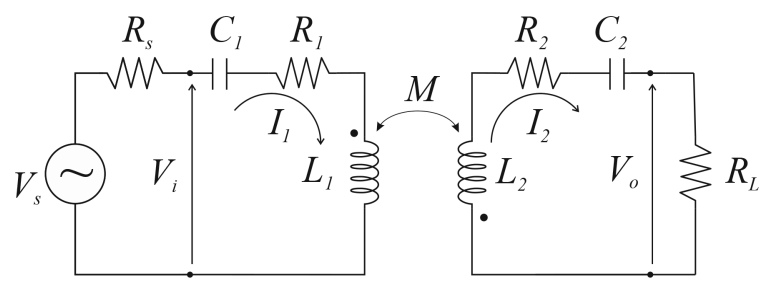

Fig. 2 Equivalent circuit model of a WPT system consisting of two resonant coils with compensation capacitances connected in series. The self- and mutual inductances calculated as shown in Section 2.2 describe the self- and mutual coupling of the transmitting and receiving coils through the conducting structure

Section 3, we investigate the impact of the conducting slab on the power transfer and the possibility of using the proposed model to compensate for the detuning effect due to the coil interaction with the slab. Results based on our analytical model regarding the conducting slab as being infinitely extended are validated through comparisons with simulations performed with CST Microwave Studio (MS) for finite width plates. Formulas for the Poynting vector and the power loss in the slab are derived in Section 4, whereby the power flow between the transmitter and receiver and the distribution of the absorption density in the slab are analysed for sample WPT configurations. Conclusions are drawn in Section 5.

\section{Theoretical formulation}

The simple WPT configuration of Fig. 1 consisting of two coaxial thin wire coils of radius $r_{0}$ above a conductive slab is considered. It is assumed that one coil (transmitter) is connected to a sinusoidal voltage source, whereas the receiver coil is connected to a load. The system usually operates at low frequencies and the two coils are inductively coupled with a coupling coefficient $k=M / \sqrt{L_{1} L_{2}}$, where $L_{n}, n=1,2$, and $M$ denote the self- and mutual inductances of the coils. To create resonant conditions, the coils are connected in series or in parallel to capacitors. In the following, we will consider the series-series compensation method.

\subsection{Coupling between two coils above a conducting slab}

The EM interaction between the wire loops can be described by means of coupled circuit theory [25] which states that the magnetic flux through each coil is related to the currents in the participating coils by

$$
\Phi_{1,2}=\int_{S_{1,2}} \boldsymbol{B}_{1,2} \times \mathrm{d} \boldsymbol{S}_{1,2}=L_{1,2} I_{1,2}+M I_{2,1}
$$

where $\boldsymbol{B}_{n}$ is the total magnetic flux density threaded with coil $n, n$ $=1,2$, as resulting from the superposition of the field contributions produced by both coil currents $I_{1}$ and $I_{2}$. Equation (1) remains valid in the presence of a conductor provided that material coupling effects are duly taken into account. The calculation of inductances $L_{1,2}$ and $M$ in the presence of the conducting slab, which are expected to generally be complex quantities [30], is detailed in the next section. Using Faraday's law of induction, with a time dependence of the form $\exp (j \omega t)$ for our quantities, where $\omega$ is the frequency, relations (1) can be rewritten in terms of the inductively induced electromotive force (EMF) in either coil as follows:

$$
\varepsilon_{1,2}=-j \omega\left(L_{1,2} I_{1,2}+M I_{2,1}\right)
$$

By taking into account ohmic losses in the coils through resistances $R_{1,2}$, that the coils are connected in series to resonance compensation capacitances $C_{1,2}$, the presence of a voltage source $V_{s}$, with internal resistance $R_{s}$, driving the transmitting coil, and the load with resistance $R_{L}$ attached to the secondary coil, (2) for the coupled coils can be recast in the form

$$
\left[\begin{array}{cc}
Z_{1}+R_{s} & j \omega M \\
j \omega M & Z_{2}+R_{L}
\end{array}\right]\left[\begin{array}{c}
I_{1} \\
I_{2}
\end{array}\right]=\left[\begin{array}{c}
V_{s} \\
0
\end{array}\right]
$$

where $Z_{1,2}$ are the total self-impedances of the primary and secondary coils

$$
Z_{n}=R_{n}+j \omega L_{n}+\frac{1}{j \omega C_{n}}, \quad n=1,2
$$

which include the ohmic resistances of the coils $R_{n}$. System of equations (3) corresponds to the equivalent circuit shown in Fig. 2. The topology of this circuit is standard for coupled coils with series-series compensation, but based on the proposed approach the physics of coil interaction mediated by the presence of the conducting slab is incorporated in the circuit response through the self- and mutual inductances (see Section 2.2). Solution of (3) for the currents $\left[I_{1}, I_{2}\right]$ reads as

$$
\begin{aligned}
& I_{1}=\frac{V_{s}}{Z_{1}+R_{s}+\omega^{2} M^{2} /\left(Z_{2}+R_{L}\right)} \\
& I_{2}=\frac{j \omega M V_{s} /\left(Z_{1}+R_{s}\right)}{\left(Z_{2}+R_{L}\right)+\omega^{2} M^{2} /\left(Z_{1}+R_{s}\right)}
\end{aligned}
$$

Then, the output power at the load can be simply calculated as

$$
P_{\text {out }}=\frac{1}{2} R_{L}\left|I_{2}\right|^{2}=\frac{1}{2} \frac{\omega^{2}|M|^{2} V_{s}^{2} R_{L}}{\left|\left(Z_{1}+R_{s}\right)\left(Z_{2}+R_{L}\right)+\omega^{2} M^{2}\right|^{2}}
$$

Concerning the PTE, its general expression is given by

$$
\eta=\eta_{p} \eta_{s}
$$

where $\eta_{p}$ and $\eta_{s}$ are the efficiency of the primary and secondary sides, respectively. The former is equal to

$$
\eta_{p}=\frac{\operatorname{Re}\left[Z_{\mathrm{ref}}^{21}\right]}{\operatorname{Re}\left[Z_{\mathrm{ref}}^{21}\right]+R_{1}}
$$

where $Z_{\text {ref }}^{21}$ represents the loading effect of the secondary on the primary circuit and is usually referred to as a reflected impedance dependent on the transformer coupling and operating frequency as

$$
Z_{\mathrm{ref}}^{21}=\frac{\omega^{2} M^{2}}{Z_{2}+R_{L}}
$$

The efficiency of the secondary circuit instead has the simple expression

$$
\eta_{s}=R_{L}\left(R_{2}+R_{L}\right)^{-1}
$$

because the current flowing through the load is the same as the current in the secondary coil. It can be shown that the highest PTE 
across weakly coupled coil links can be achieved when both $L C$ resonators are tuned at the same resonance frequency, i.e. when $\omega_{1}=1 / \sqrt{\operatorname{Re}\left[L_{1}\right] C_{1}}=\omega_{2}=1 / \sqrt{\operatorname{Re}}\left[L_{2}\right] C_{2}=\omega_{0}$ [24]. Typically, the coils are small with respect to the wavelength and the selfcapacitance is negligible compared to the required tuning capacitor for resonance at low frequency. Therefore, the required tuning capacitances of the coils, which are assumed to be lossless, can be simply defined in terms of the coil self-inductances and resonant frequency as

$$
C_{1,2}=\frac{1}{\omega_{0}^{2} \operatorname{Re}\left[L_{1,2}\right]}
$$

\subsection{Calculation of self- and mutual inductances}

The determination of the currents in the coupled coils requires the knowledge of the self-inductances of the transmitting and receiving coils and of the mutual inductance $M$ in the presence of the conducting slab. As mentioned above, these quantities can be calculated based on the analytical solution developed in [29]. If the contribution to the total magnetic flux density $\boldsymbol{B}_{n}$ arising from each coil is written as the curl of the vector potential associated with the respective current $\boldsymbol{A}_{m}$, in accordance with Stokes' theorem the surface integral in (1) can be recast into a contour integral so that the EMFs in the coils read as

$$
\begin{aligned}
\varepsilon_{1,2} & =-j \omega \int_{C_{1,2}} \boldsymbol{A}_{1,2} \times \mathrm{d} \boldsymbol{C}_{1,2} \\
& =-j \omega\left(I_{1,2} \int_{C_{1,2}} \boldsymbol{A}_{11,22} \times \mathrm{d} \boldsymbol{C}_{1,2}+I_{2,1} \int_{C_{1,2}} \boldsymbol{A}_{12,21} \times \mathrm{d} \boldsymbol{C}_{1,2}\right)
\end{aligned}
$$

where $\boldsymbol{A}_{\mathrm{nm}}, n, m=1,2$, denotes the vector potential created by a unit current flowing in the $m$ th coil at the position of the $n$th one. By comparing (12) with (2), self- and mutual inductances can be generally expressed in the magnetic vector potential formalism as

$$
\begin{aligned}
L_{1,2} & =\int_{C_{1,2}} \boldsymbol{A}_{11,22} \times \mathrm{d} \boldsymbol{C}_{1,2} \\
M & =\int_{C_{1}} \boldsymbol{A}_{12} \times \mathrm{d} \boldsymbol{C}_{1}=\int_{C_{2}} \boldsymbol{A}_{21} \times \mathrm{d} \boldsymbol{C}_{1}
\end{aligned}
$$

In particular, taking advantage of the circular symmetry of our problem

$$
\begin{aligned}
L_{1,2} & =2 \pi r_{0} \boldsymbol{A}_{11,22} \\
M & =2 \pi r_{0} \boldsymbol{A}_{12}=2 \pi r_{0} \boldsymbol{A}_{21}
\end{aligned}
$$

Due to axial symmetry, the vector potential has only a component along the azimuthal angle $\phi$, i.e. $\boldsymbol{A}_{\mathrm{nm}}=A_{\phi}^{(i)}\left(r_{0}, h_{n}, h_{m}\right) \hat{\phi}$, and its exact representation can be found in the form of integrals of firstorder Bessel functions as according to the solution in [29]

$$
A_{\phi}^{(i)}(r, z, h)=\mu_{0} r_{0} \int_{0}^{\infty} J_{1}\left(\alpha r_{0}\right) J_{1}(\alpha r) F^{(i)}(\alpha, z, h) \alpha \mathrm{d} \alpha
$$

In (15), the cylindrical coordinates $(r, z)$ identify the field point, $h$ is the height of the coil over the conducting interface, whose upper face is assumed to be located at $z=0$, and the index $i, i=1,2,3,4$, refers to the different regions, shown in Fig. 3, in which the problem is divided, i.e. above the reference coil $(i=1)$, below the coil but above the conductor $(i=2)$, in the conductor slab $(i=3)$, and in the free space below the conductor $(i=4)$. For the single conducting slab configuration considered in this work, which is a special case of that analysed in [29] with the medium in region 4 taken as air instead that a conducting half space, the spectral functions $F_{i}$ can be obtained from the continuity of the fields at the various interfaces like in [29] and read as

$$
\begin{aligned}
& F^{(1)}=\frac{e^{-\alpha(z-h)}}{2 \alpha}+\frac{\left(\alpha-\alpha_{1}\right)\left(\alpha+\alpha_{1}\right)\left(e^{2 \alpha_{1} t}-1\right)}{\left(\alpha+\alpha_{1}\right)^{2} e^{2 \alpha_{1} t}-\left(\alpha-\alpha_{1}\right)^{2}} \frac{e^{-\alpha(z+h)}}{2 \alpha} \\
& F^{(2)}=\frac{e^{-\alpha(h-z)}}{2 \alpha}+\frac{\left(\alpha-\alpha_{1}\right)\left(\alpha+\alpha_{1}\right)\left(e^{2 \alpha_{1} t}-1\right)}{\left(\alpha+\alpha_{1}\right)^{2} e^{2 \alpha_{1} t}-\left(\alpha-\alpha_{1}\right)^{2}} \frac{e^{-\alpha(h+z)}}{2 \alpha} \\
& F^{(3)}=\mathrm{e}^{-\alpha h} \frac{\left(\alpha+\alpha_{1}\right) e^{2 \alpha_{1} t} e^{\alpha_{1} z}+\left(\alpha_{1}-\alpha_{0}\right) e^{-\alpha_{1} z}}{\left(\alpha+\alpha_{1}\right)^{2} e^{2 \alpha_{1} t}-\left(\alpha-\alpha_{1}\right)^{2}} \\
& F^{(4)}=\mathrm{e}^{-\alpha h} \frac{2 \alpha_{1} e^{\left(\alpha+\alpha_{1}\right) t} e^{\alpha z}}{\left(\alpha+\alpha_{1}\right)^{2} e^{2 \alpha_{1} t}-\left(\alpha-\alpha_{1}\right)^{2}}
\end{aligned}
$$

In (16), the quantity $\alpha_{1}$, introduced in the derivation of the solution by the method of separation of variables, contains the dependence on the conductivity $\sigma$ of the slab: $\alpha_{1}=\sqrt{\alpha^{2}+j \omega \mu_{0} \sigma_{1}}$. The specific function $F^{(i)}$ to be used in (15) for calculating (14) depends on the position of the pickup coil.

Incorporating (14) into the equivalent circuit model presented in the previous section, particularly (5), provides exact expressions for the currents in the coils and all other quantities of interest, e.g. output power (6) and PTE (7), which account for all the EM interactions arising in the inductively coupled system in the presence of a conductor. In particular, feedback effects from the receiving to the transmitting coil and material coupling effects of the receiving coil, which were not considered in original eddycurrent models [29], are completely addressed by the proposed approach.

\section{Optimal power transfer between two coils in the presence of a conducting slab}

Both the resonance frequency and the quality factor of the resonators are influenced by the environment. As a consequence, the performance of the WPT system will change as well. In particular, the close proximity of a conducting plate will greatly influence the power transfer system. We demonstrate in this section that the proposed model can be instrumental in designing the optimal WPT regime for the illustrative case of two coils above a conducting slab. Throughout all results presented in the following, the coil configuration is kept the same: the two single turn coils have a radius $r_{0}=11 \mathrm{~mm}$ and are spaced apart a distance $d=10 \mathrm{~mm}$. Without loss of generality due to the standard frequency scaling of EM phenomena, a sample operating frequency $\omega_{0}=10^{9} \mathrm{rad} / \mathrm{s}$, i.e. $f=159 \mathrm{MHz}$, is assumed at which the coil electrical size is sufficiently small to render their radiation losses negligible.

First, we consider the WPT system made of the two coaxial coils in free space. One of the coils is driven by a voltage source $V_{s}=10$ with internal resistance $R_{s}=5$. Output power can be maximised by tuning the coils to resonate at the same frequency, maximising the coupling by orienting the loops in a suitable manner, for example as in our coaxial configuration, and choosing an optimal load resistance $R_{\mathrm{L}}$. In particular, the coils are made resonant at the desired frequency by loading them with series compensation capacitances which are determined with (11). From

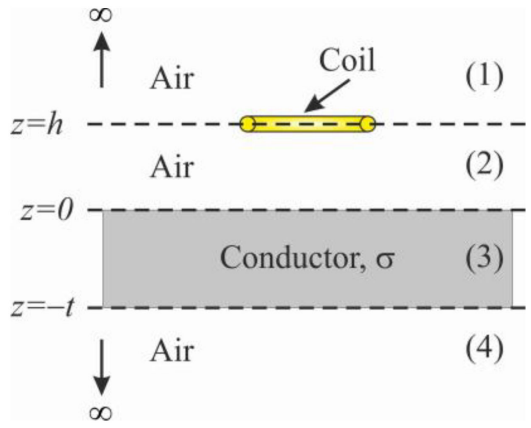

Fig. 3 Layered space structure used to find the field produced by a coil located at a certain distance $(z=h)$ above a conducting layer with thickness $t$ [29] 
the plot of the output power in free space as a function of the load resistance, represented by the blue curve in Fig. 4, it can be seen that a suitable value of $R_{\mathrm{L}}$ can be chosen to maximise $P_{\text {out }}$. Fullwave simulations performed with CST MS, shown as the blue circles in Fig. 4, confirm the results of the theory.

In Fig. 4, the effect of introducing a conducting slab below the two coaxial coils is also analysed. The two coils are parallel to the slab and lie on the same side of it, as shown in Fig. 1. The lower transmitting coil is located at $h_{1}=2 \mathrm{~mm}$ above the conducting slab. It is assumed that the slab is 5 -mm-thick and its conductivity is $\sigma=100 \mathrm{~S} / \mathrm{m}$. The output power at the receiving coil when the coils are loaded with the same compensation capacitances selected for the free space case appears to be significantly reduced. This is not only due to the inevitable losses in the conducting slab; indeed, the interaction with the slab changes the self-impedances of the coils, and since this effect is stronger on the coil closer to the slab, the coils turn out to be detuned and resonate at different frequencies, which contributes to decreasing the transferred power. However, these variations of the self- and mutual impedances can be predicted with the model proposed in this work, which in turn allows one to choose appropriately distinct values for the compensation capacitances to tune the resonators to resonate at the same resonance frequency also in the presence of the conducting slab. By doing so, a significantly larger output power can be obtained with the optimal load resistance, as illustrated by the red curve in Fig. 4 that corresponds to the received power in the retuned system. To validate the theoretical predictions, also these configurations has been simulated with CST MS, using the same compensation capacitances inserted in the coils, made of a round wire with diameter of $1 \mathrm{~mm}$, and modelling the slab as a finite circular plate with radius of $50 \mathrm{~mm}$. Simulated data, plotted with markers in Fig. 4, are found to be in very good agreement with analytical results for both the 'detuned' and 'retuned' scenarios, which proves the effectiveness and accuracy of the model.

Our method can actually be used to estimate the detuning effect of the conducting slab for arbitrary geometrical and electrical parameters of the system. As an example, in Fig. 5 are shown the maximum output power and the optimal load resistance for our reference coil configuration above a conducting slab of variable conductivity. The lower coil, at $h_{1}=2 \mathrm{~mm}$ over the slab, is fed by a voltage source $V_{s}=10$ with resistance $R_{s}=5$. Again, the received power is reported both in the case in which the compensation capacitances are chosen once and for all for $\sigma=0$ (dashed red curve), and when the proposed model is used to retune the coils for each conductivity value (solid red curve). The performance improvement in the latter case is evident, especially for larger slab conductivities. However, it is noted that as $\sigma$ rises the optimal value of $R_{L}$ (solid blue curve) tends to fall to increasingly small values that can become unmanageable if $\mathrm{AC}$ to $\mathrm{DC}$ rectification is required at the output circuit. In Fig. 5, are also shown the data retrieved by full-wave simulations of the WPT system for a few sample values of conductivity of a finite conducting plate with radius of $50 \mathrm{~mm}$, which appear to be practically superimposed with the curves of the analytical results.

\section{Power flow and slab absorption}

One of the most notable advantages of the model presented in this work is that it allows framing the description of WPT in a complex environment, possibly including multiple conducting interfaces, within the same simple equivalent circuit formalism used for WPT in free space; as a result, the main parameters characterising the power link, e.g. the output power (6) and transfer efficiency (7), can be readily computed, while all relevant EM effects and interaction mechanisms are subsumed into the calculation of the inductances. Yet, the model can provide additional and complementary information with respect to pure lumped circuit theory. In fact, once the currents in the coils are obtained by solving the coupled circuits (3), the electric and magnetic field vectors can be determined; and from the knowledge of the fields, the Poynting vector at any point can be also derived, which can provide a comprehensive understanding of the energy transfer mechanism involved in the WPT system. In each region into which the problem is subdivided (cf. Fig. 3), the Poynting vector can be computed as

$$
\boldsymbol{S}^{(i)}=\frac{1}{2} \boldsymbol{E}^{(i)} \times \boldsymbol{H}^{(i)^{*}}
$$

where $i=1,2,3,4$, and the electric and magnetic fields $\boldsymbol{E}^{(i)}, \boldsymbol{H}^{(i)}$ are generally given by the superposition of the fields produced by each coil:

$$
\left[\boldsymbol{E}^{(i)}, \boldsymbol{H}^{(i)}\right]=\sum_{n=1}^{2}\left[\boldsymbol{E}_{n}^{(i)}, \boldsymbol{H}_{n}^{(i)}\right]
$$

Under a magneto-quasistatic approximation, the electric and magnetic fields $\left[\boldsymbol{E}_{n}^{(i)}, \boldsymbol{H}_{n}^{(i)}\right]$ can be obtained from the corresponding vector potential (15) as

$$
\begin{aligned}
E_{\phi, n}^{(i)} & =-j \omega A_{\phi, n}^{(i)} \\
& =-j \omega \mu_{0} I_{n} r_{0} \int_{0}^{\infty} J_{1}\left(\alpha r_{0}\right) J_{1}(\alpha r) F^{(i)}\left(\alpha, z, h_{n}\right) \alpha \mathrm{d} \alpha, \\
H_{r, n}^{(i)} & =-\frac{1}{\mu_{0}} \frac{\partial A_{\phi, n}^{(i)}}{\partial z} \\
& =-I_{n} r_{0} \int_{0}^{\infty} J_{1}\left(\alpha r_{0}\right) J_{1}(\alpha r) \frac{\partial F^{(i)}\left(\alpha, z, h_{n}\right)}{\partial z} \alpha \mathrm{d} \alpha, \\
H_{z, n}^{(i)} & =\frac{1}{\mu_{0}} \frac{1}{r} \frac{\partial r A_{\phi, n}^{(i)}}{\partial r} \\
& =I_{n} r_{0} \int_{0}^{\infty} J_{1}\left(\alpha r_{0}\right) J_{0}(\alpha r) F^{(i)}\left(\alpha, z, h_{n}\right) \alpha^{2} \mathrm{~d} \alpha,
\end{aligned}
$$

where $n=1,2$.

The model can constitute an effective tool for the fast exploration of the power flow and distribution between the transmitting and receiving coils of a WPT system in the presence of a conducting medium, as well as for estimating the level of absorption within it and localising possible hot spots and the associated heat generation. These aspects are particularly important for wirelessly powered implanted medical devices and body sensor networks to verify that the basic restrictions for human exposure defined in terms of specific absorption rate (SAR), induced currents, and electric fields are not violated [22]. To estimate the total absorption loss in the conducting material, we can either consider the power flow through the upper face of the slab along the $(-\hat{z})$ direction, whose real part, based on the conservation of energy in the considered WPT systems, corresponds to the power loss in the slab; or, equivalently, we can integrate the electric power loss density $\boldsymbol{J}^{(3)} \cdot \boldsymbol{E}^{(3)}$ over the volume of the slab, with $\boldsymbol{J}^{(3)}=\sigma \boldsymbol{E}^{(3)}$ denoting the eddy-current density within it. Following the latter approach and taking into account (18), we can write the slab absorption in the EM form

$$
A_{\text {slab }}^{\mathrm{EM}}=\frac{1}{2} \sigma \int_{V}\left\{\sum_{n=1}^{2}\left[\boldsymbol{E}_{n}^{(3)} \times \boldsymbol{E}_{n}^{(3)^{*}}\right]+2 \operatorname{Re}\left[\boldsymbol{E}_{1}^{(3)} \times \boldsymbol{E}_{2}^{(3)^{*}}\right]\right\} d V
$$

Inserting the expressions (19) of the fields into (20), introducing the function $F^{\prime}(\alpha, z)$ such that $F^{(3)}\left(\alpha, z, h_{n}\right)=F^{\prime}(\alpha, z) e^{-\alpha h_{n}}$, and making use of the Fourier-Bessel integral [32], as done in [29] to simplify the relations descending from the boundary conditions, (20) can be rewritten as

$$
\begin{aligned}
& A_{\text {slab }}^{\mathrm{EM}}=\pi \sigma\left(\omega \mu_{0} r\right)^{2} \int_{-c}^{0} \int_{0}^{\infty}\left[J_{1}\left(\alpha r_{0}\right)\right]^{2}\left|F^{\prime}{ }_{3}(\alpha, z)\right|^{2} . \\
& \left\{\left|I_{1}\right|^{2} e^{-2 \alpha h_{1}}+\left|I_{2}\right|^{2} e^{-2 \alpha h_{2}}+2 \operatorname{Re}\left[I_{1} I_{2}^{*}\right] e^{-\alpha\left(h_{1}+h_{2}\right)}\right\} \alpha \mathrm{d} \alpha \mathrm{d} z
\end{aligned}
$$

Equation (21) clearly highlights that absorption in the slab arises as the effect of three contributions corresponding to the interaction of 


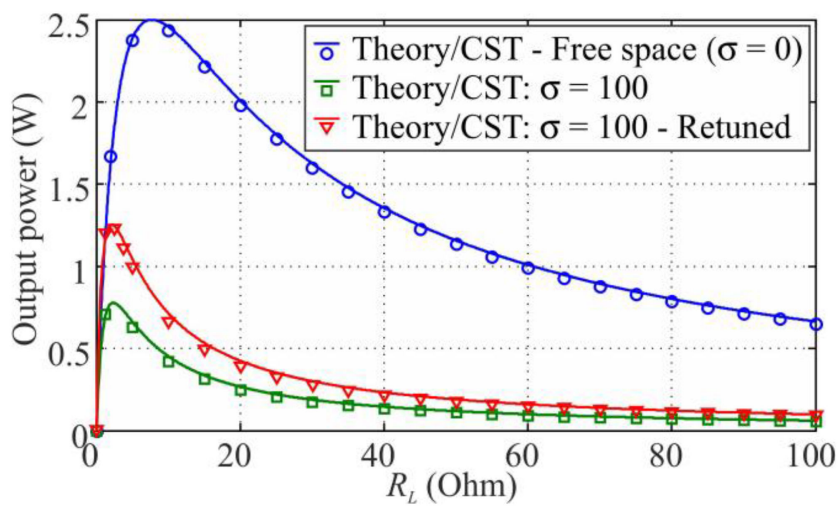

Fig. 4 WPT between two coils in free space (blue line and markers) and when placed above a 5-mm-thick conducting slab with conductivity $\sigma=100$ S/ $m$ (green and red lines and markers) at the operating frequency $\omega_{0}=10^{9} \mathrm{rad} / \mathrm{s}$. The coils have radius $r_{0}=11 \mathrm{~mm}$ and are a distance $d=10 \mathrm{~mm}$ apart. The lower coil located at $h_{1}=2 \mathrm{~mm}$ above the conducting slab is the transmitter. Results of the analytical model assuming an infinitely wide slab, plotted as lines, are compared with simulated data obtained with CST MS for a finite circular plate with radius of 50 mm and a coil wire diameter of 1 mm, shown with markers. The output power in the presence of the slab is shown both when the compensation capacitances are kept the same as in free space (green line and square markers) and when the coils are retuned based on the predictions of the proposed model (red line and triangular markers)

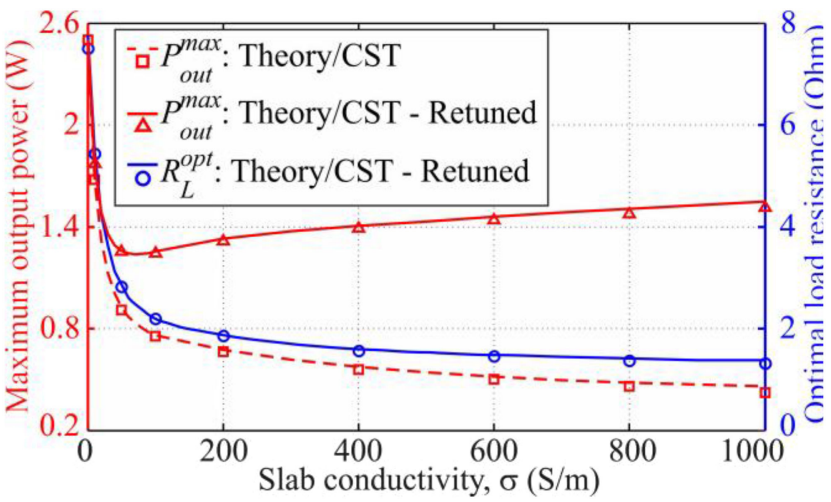

Fig. 5 Maximum output power (solid and dashed red lines and square and triangular markers) and optimal loading resistance (blue line and circles) for the reference WPT system of two coils in the presence of a 5-mm thick conducting slab of variable conductivityo. Solid lines correspond to the analytical results, regarding the slab as being infinitely wide, whereas markers are data obtained with full-wave simulations with CST MS for a finite circular plate with radius of $50 \mathrm{~mm}$. The maximum output power is shown both when the compensation capacitances are kept the same as in free space, i.e. for $\sigma=0$ (dashed red line and square markers), and when the coils are retuned in accordance with predictions of the proposed model (solid red line and triangular markers)

the eddy currents induced in the slab with those fractions of the fields produced by the transmitting and receiving coils and their cross-coupling mediated through the slab. Importantly, unless the receiving coil is open-circuited and $I_{2}=0$, it would be incorrect to neglect the latter two terms of (21) in the calculation of the absorption, and an analogous consideration applies to the power flow.

It is interesting to compare (21) with the expression of the power loss deduced in terms of circuit quantities; the total active power absorbed by the WPT system can be expressed by the following generalisation of the standard power relation for a single lumped element to the case of a multi-port circuit

$$
P=\frac{1}{2} \operatorname{Re}\left[\bar{V}^{T} \bar{I}\right] *=\frac{1}{2} \operatorname{Re}\left[\bar{I}^{T} \overline{\bar{Z}}^{T} \bar{I}^{*}\right]
$$

where $\overline{\bar{Z}}$ denotes the $2 \times 2$ impedance matrix introduced in (3), $\bar{V}=\left[V_{s}, 0\right]$ and $\bar{I}=\left[I_{1}, I_{2}\right]$ are the vectors of the voltages and currents at the circuit ports, and the vector form $\overline{\bar{Z}} \bar{I}=\bar{V}$ of (3) has been used. Performing the multiplications in (22) and discarding those terms representing the ohmic losses in the coils and the output power, the absorption in the conducting slab can be cast solely in terms of the circuit (CKT) parameters as

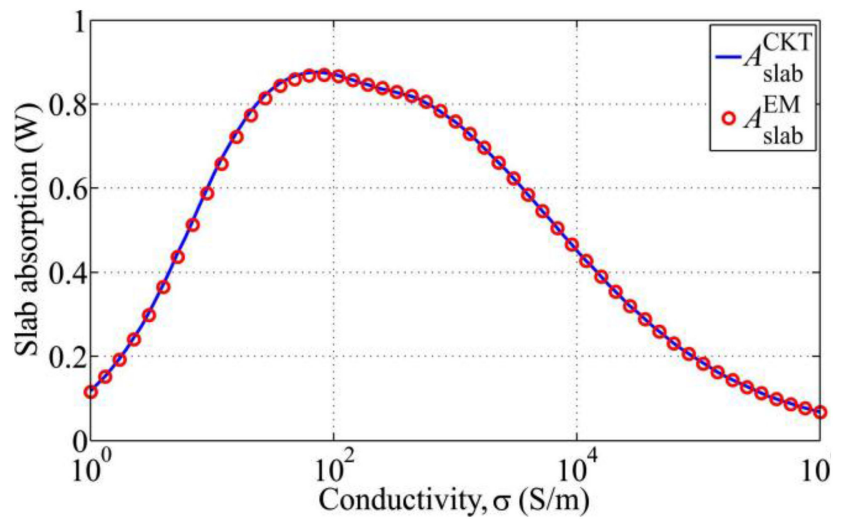

Fig. 6 Absorption produced in a 5-mm-thick conducting slab of variable conductivity $\sigma$ by the WPT system formed by two resonant circular coils with radius $r_{0}=11 \mathrm{~mm}$ and spaced apart a distance $d=10 \mathrm{~mm}$, operating at the frequency $\omega_{0}=10^{9} \mathrm{rad} / \mathrm{s}$. The lower coil, acting as the transmitter, is located at $h_{1}=2 \mathrm{~mm}$ above the conducting slab. The absorption is shown as calculated with both the EM field-and circuit-based expressions (21) and (23) (solid blue line and red circles, respectively)

$$
\begin{aligned}
A_{\text {slab }}^{\mathrm{CKT}}= & -\frac{1}{2}\left\{\omega \operatorname{Im}\left[L_{1}\right]\left|I_{1}\right|^{2}+\omega \operatorname{Im}\left[L_{2}\right]\left|I_{2}\right|^{2}\right. \\
& \left.+2 \omega \operatorname{Im}[M] \operatorname{Re}\left[I_{1} I_{2}^{*}\right]\right\}
\end{aligned}
$$

(note that the imaginary parts of $L_{1,2}$ and $M$ are negative with the assumed time dependence). The EM field- and circuit-based expressions of the slab absorption (21) and (23) are shown in Fig. 6 for the same sample two coils WPT system operating at the fixed frequency $\omega_{0}=10^{9} \mathrm{rad} / \mathrm{s}$ considered throughout the paper, with the lower transmitting coil placed at $2 \mathrm{~mm}$ above a 5 -mm-thick conducting slab of variable conductivity. As apparent, (21) and (23) provide identical results, which further confirms the capability of the proposed generalised equivalent circuit model of incorporating all EM interactions occurring between the coils and the slab. Moreover, this comparison clarifies that the self- and mutual inductances of inductively coupled coils in the presence of a conductor become complex quantities, as their imaginary parts are obviously proportional to resistive terms accounting for the losses associated with the energy dissipated as heat by the respective fractions of the eddy currents induced in a conducting material with finite conductivity.

For the purpose of illustration, we show in Fig. 7 the magnitude of the power flow density and direction of the Poynting vector $S$ from the transmitting coil, at the bottom, to the receiving coil, at the top, in the mid transverse cross-section of the sample WPT system formed by two 11-mm-radius, $10-\mathrm{mm}$-spaced apart single 

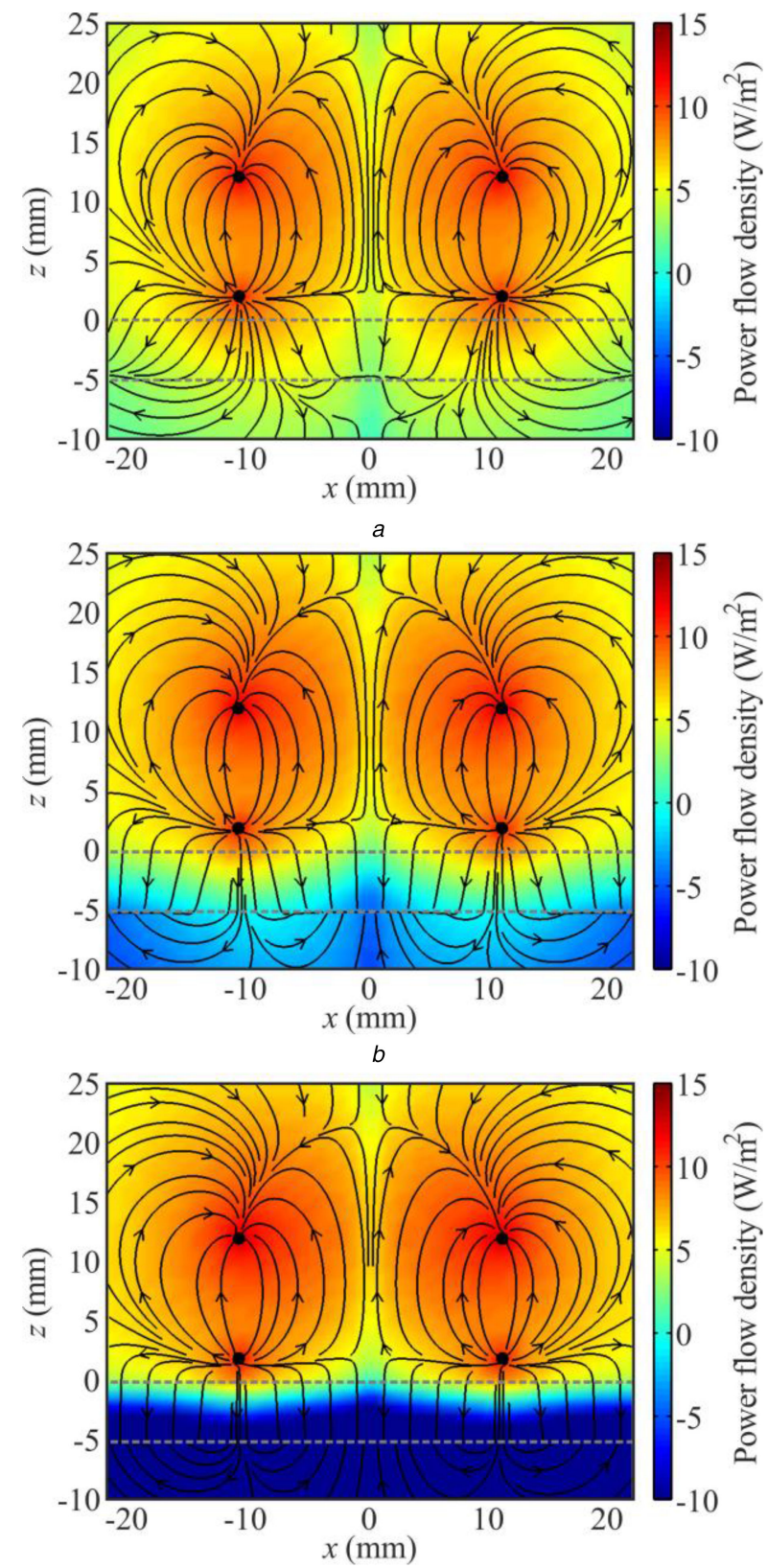

c

Fig. 7 Power flow from transmitter to receiver in the mid transverse cross sectional plane of the WPT system formed by two resonant coils operating at the frequency $\omega_{0}=10^{9} \mathrm{rad} / \mathrm{s}$ in the presence of a 5-mm-thick conducting slab of variable conductivity $\left(\sigma=10^{2}, 10^{3}, 10^{4} \mathrm{~S} / \mathrm{m}\right)$. The coils have radius $r_{0}=11 \mathrm{~mm}$ and are a distance $d=10 \mathrm{~mm}$ apart. The lower coil, acting as the transmitter, is located at $h_{1}=2 \mathrm{~mm}$ above the conducting slab

(a) $\sigma=10^{2}$, (b) $\sigma=10^{3}$, and (c) $\sigma=10^{4} \mathrm{~S} / \mathrm{m}$. Small black circles indicate the positions of the coils, whereas the dashed grey lines correspond to the slab interfaces

turn coils with a 5-mm-thick conductive slab lying $2 \mathrm{~mm}$ below the transmitting coil. The frequency of operation is again $\omega_{0}=10^{9}$ $\mathrm{rad} / \mathrm{s}$ and three different values of slab conductivity, $\sigma=10^{2}, 10^{3}, 10^{4} \mathrm{~S} / \mathrm{m}$, are considered. The corresponding maps of the absorption density $\sigma|\boldsymbol{E}|^{2} / 2$ are presented in Fig. 8. These maps are computed by (17)-(19) along with (16). For all values of conductivity analysed, it can be observed in Fig. 7 that the slab introduces a significant modification and redirection of the power flow, both in the lower half space, which is progressively screened from the fields for increasing slab conductivity, and between the two coils, as a consequence of the Poynting vector being diverted to be substantially perpendicular to the conducting interfaces by the boundary conditions there constraining the fields. Meanwhile, Fig. 8 demonstrates that strong and diffused absorption occurs in the slab for both $\sigma=10^{2}$ and $\sigma=10^{3} \mathrm{~S} / \mathrm{m}$, though in a gradually

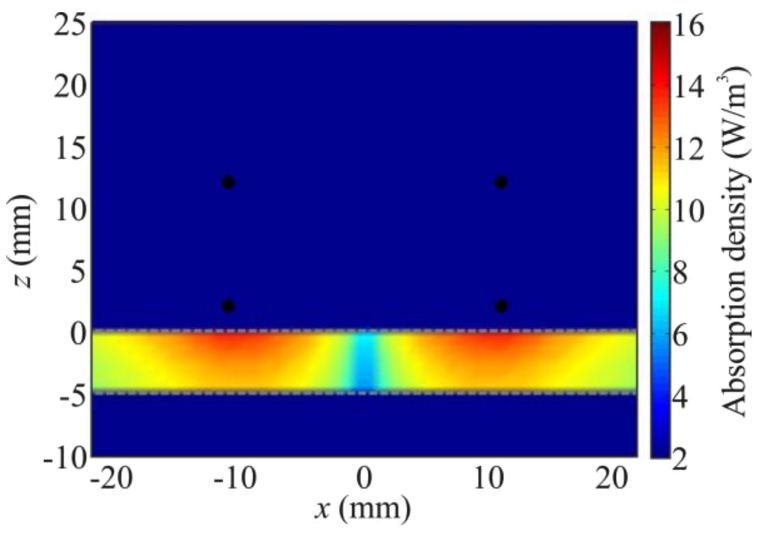

a

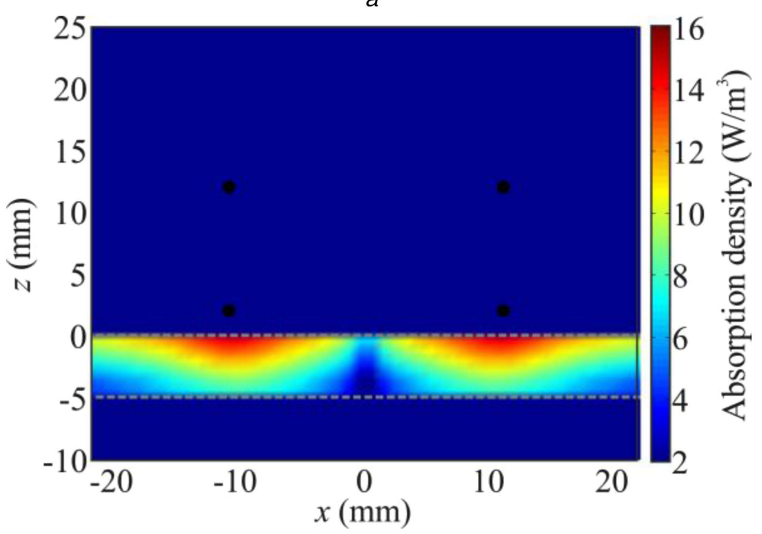

$b$

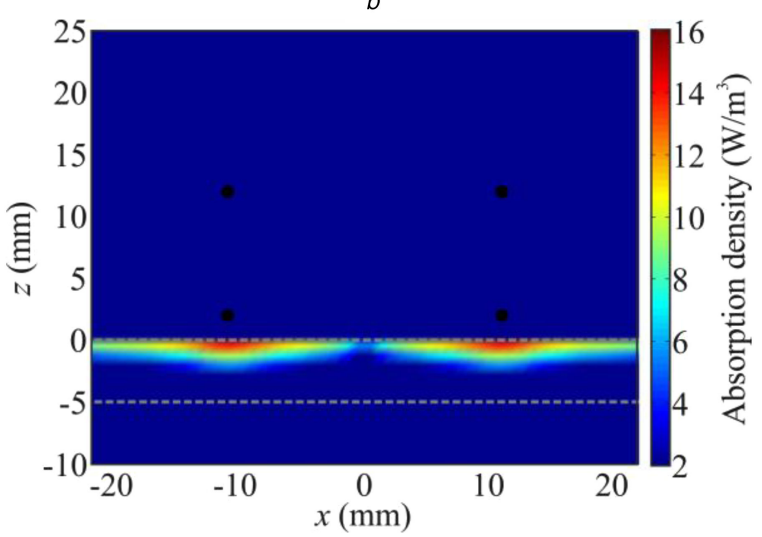

$c$

Fig. 8 Absorption density in the mid transverse cross sectional plane of the same WPT system for which power flow is shown in Fig. 7. Conductivity of the 5-mm-thick slab below the coils is variable as (a) $\sigma=10^{2}$, (b) $\sigma=10^{3}$, and (c) $\sigma=10^{4} \mathrm{~S} / \mathrm{m}$

decreasing thickness; instead for $\sigma=10^{4} \mathrm{~S} / \mathrm{m}$ there is little energy being absorbed by the eddy-current losses in the slab, which are confined in a very shallow layer at the surface; the trend seen is indeed consistent with skin effect considerations and the curve of the overall slab absorption as a function of conductivity shown in Fig. 6. It is noted that SAR information for assessment of RF exposure could be readily deduced from the total absorption or its local spatial distribution based on the additional knowledge of the material density.

\section{Conclusions}

An analytical approach to characterise the WPT between two coils in the presence of a conducting slab has been developed. EM theory and an equivalent coupled circuit model have been combined to achieve a comprehensive description of this system that accounts for all the interactions arising between the magnetically coupled coils and through the conductor. Feedback effects from the receiving to the transmitting coil and material coupling effects of the receiving coil, which were not considered in 
original eddy current models, are completely addressed by the proposed method. It has been shown that this approach permits to analytically optimise the power transfer between the coils by predicting the different detuning effect that the conducting slab has on the coils depending on their position. While the model has been presented for simplicity for the case of a WPT configuration formed by two identical coaxial single turn coils, it can be straightforwardly generalised to describe systems comprising an arbitrary number of diverse resonators with finite rectangular cross-sections placed in arbitrary positions, i.e. not necessarily coaxially aligned, and more complex multi-layered conducting and ferromagnetic structures. Moreover, the effect of the finite extension of the conducting slab could be explicitly taken into account by exploiting the technique for calculating the eddy current distribution in thin conductive plates due to an arbitrary magnetic source developed in [33]. Comparisons with results obtained by full-wave numerical simulations have been provided to confirm the validity of the proposed model. As an additional benefit, the model allows fast estimation of the power flow and localisation of absorption peaks, offering unique insight into the physics and operation of a WPT system in a complex environment, which can have crucial relevance in the design and deployment of wirelessly fed bio-medical devices and body sensor networks.

\section{Acknowledgments}

Partial support for this work was provided by the UK Engineering and Physical Science Research Council (EPSRC) under the project 'Synthesizing 3D Metamaterials for RF, Microwave and $\mathrm{THz}$ Applications' (grant EP/N010493/1). Fruitful discussions with Prof. Lazslo Solymar, Imperial College, London, are thankfully acknowledged.

\section{References}

[1] Tesla, N.: 'The transmission of electric energy without wires', Electr. World Eng., 1904, 5, pp. 162-167

[2] Tesla, N.: 'The transmission of electrical energy without wires as a means of furthering peace', Electr. World Eng., 1905, 45, (1), pp. 21-24

[3] Kurs, A., Karalis, A., Moffatt, R., et al.: 'Wireless power transfer via strongly coupled magnetic resonances', Science, 2007, 317, (5834), pp. 83-86

[4] Cheon, S., Kim, Y.H., Kang, S.Y., et al.: 'Circuit-model-based analysis of a wireless energy-transfer system via coupled magnetic resonances', IEEE Trans. Ind. Electron., 2011, 58, (7), pp. 2906-2914

[5] Kiani, M., Ghovanloo, M.: 'The circuit theory behind coupled-mode magnetic resonance-based wireless power transmission', IEEE Trans. Circuits Syst.Part I, 2012, 59, (8), pp. 1-10

[6] Kim, J.W., Son, H.C., Kim, K.H., et al.: 'Efficiency analysis of magnetic resonance wireless power transfer with intermediate resonant coil', IEEE Antennas Wirel. Propag. Lett., 2011, 10, pp. 389-392

[7] Kiani, M., Jow, U.-M., Ghovanloo, M.: 'Design and optimization of a 3-coil inductive link for efficient wireless power transmission', IEEE Trans. Biomed. Circuits Syst., 2011, 5, (6), pp. 579-591

[8] Hui, S.Y.R., Zhong, W., Lee, C.K.: 'A critical review of recent progress in mid-range wireless power transfer', IEEE Trans. Power Electron., 2014, 29, (9), pp. 4500-4511

[9] Choi, J., Seo, C.H.: 'High-efficiency wireless energy transmission using magnetic resonance based on negative refractive index metamaterial', Prog. Electromagn. Res., 2010, 106, pp. 33-47
[10] Wang, B., Teo, K.H., Nishino, T., et al.: 'Experiments on wireless power transfer with metamaterials', Appl. Phys. Lett., 2011, 98, (25), p. 254101

[11] Urzhumov, Y., Smith, D.R.: 'Metamaterial-enhanced coupling between magnetic dipoles for efficient wireless power transfer', Phys. Rev. B, 2011, 83, (20), pp. 1-10

[12] Stevens, C.J.: 'Power transfer via metamaterials', Comput. Mater. Contin, 2013, 33, (1), pp. 1-18

[13] Stevens, C.J.: 'Magnetoinductive waves and wireless power transfer', IEEE Trans. Power Electron., 2015, 30, (11), pp. 6182-6190

[14] Sample, A.P., Meyer, D.T., Smith, J.R.: 'Analysis, experimental results, and range adaptation of magnetically coupled resonators for wireless power transfer', IEEE Trans. Ind. Electron., 2011, 58, (2), pp. 544-554

[15] Vilathgamuwa, D., Sampath, J.: 'Wireless power transfer (WPT) for electric vehicles (EVs) - present and future trends', in Rajakaruna, S., Shahnia, F., Ghosh, A. (Eds.): 'Plug in electric vehicles in smart grids' (Springer, Berlin, Germany, 2015), Chap. 2, pp. 33-60

[16] Baker, M.W., Sarpeshkar, R.: 'Feedback analysis and design of RF power links for low-power bionic systems', IEEE Trans. Biomed. Circuits Syst., 2007, 1, (1), pp. 28-38

[17] RamRakhyani, A.K., Mirabbasi, S., Chiao, M.: 'Design and optimization of resonance-based efficient wireless power delivery systems for biomedical implants', IEEE Trans. Biomed. Circuits Syst., 2011, 5, (1), pp. 48-63

[18] Ho, J.S., Kim, S., Poon, A.S.Y.: 'Midfield wireless powering for implantable systems', Proc. IEEE, 2013, 101, (6), pp. 1369-1378

[19] Kong, F., Huang, Y., Najafizadeh, L.: 'A coil misalignment compensation concept for wireless power transfer links in biomedical implants'. 2015 IEEE Wireless Power Transfer Conf. (WPTC), Boulder, CO, USA, May 2015, pp. $1-4$

[20] Rao, S., Chiao, J.C.: 'Body electric: wireless power transfer for implant applications', IEEE Microw. Mag., 2015, 16, (2), pp. 54-64

[21] Zhang, F., Liu, J., Mao, Z., et al.: 'Mid-range wireless power transfer and its application to body sensor networks', Open J. Appl. Sci., 2012, 2, pp. 35-46

[22] Christ, A., Douglas, M.G., Roman, J.M., et al.: 'Evaluation of wireless resonant power transfer systems with human electromagnetic exposure limits', IEEE Trans. Electromagn. Compat., 2013, 55, (2), pp. 265-274

[23] Yu, X., Sandhu, S., Beiker, S., et al.: 'Wireless energy transfer with the presence of metallic planes', Appl. Phys. Lett., 2011, 99, pp. 1-3

[24] Yu, X., Skauli, T., Skauli, B., et al.: 'Wireless power transfer in the presence of metallic plates: experimental results', AIP. Adv., 2013, 3, pp. 1-9

[25] Fano, R.M., Chu, L.J., Adler, R.B.: 'Electromagnetic fields, energy and forces' (Wiley, New York, NY, USA, 1960)

[26] Hammond, P.: 'The calculation of the magnetic field of rotating machines. Part 1.-The field of a tubular current', Proc. IEE, Monograph No. 333, 1959 , 106C, p. 158

[27] Ashworth, D.S., Hammond, P. 'The calculation of the magnetic field of rotating machines. Part 2: The field of turbo-generator end-windings', Proc. IEE., Monograph No. 3489 S, 1961, 108 A, p. 527

[28] Hammond, P.: 'The calculation of the magnetic field of rotating machines. Part 3.- - eddy currents induced in a solid slab by a circular current loop', Proc. IEE, 1962, 514S, pp. 508-515

[29] Dodd, C.V., Deeds, W.E.: 'Analytical solutions to eddy-current probe-coil problems', J. Appl. Phys., 1968, 39, (6), pp. 2829-2838

[30] Vallecchi, A., Chu, S., Solymar, L., et al.: 'Coupling between coils in the presence of conducting medium', IET Microw., Antennas Propag., 2018, 14, p. 11

[31] Vallecchi, A., Stevens, C., Shamonina, E.: 'Wireless power transfer in the presence of a conducting interface: an analytical solution'. Loughborough Antennas \& Propagation Conf. (LAPC 2017), Loughborough, 2017, pp. 1-5

[32] Watson, G.N.: 'Theory of Bessel functions' (Cambridge University Press, Cambridge, UK, 1944), p. 453

[33] Subhra, P.J., Bird, Z.: 'A 3-D analytic eddy current model for a finite width conductive plate', COMPEL: Int. J. Comput. Math. Electr. Electron. Eng., 2013, 33, (1/2), pp. 688-706 